\title{
A perspectiva da translação do conhecimento nos programas de mestrado profissional na área da enfermagem
}

\author{
The perspective of knowledge translation in professional master's programs in nursing \\ La perspectiva de la traducción del conocimiento en los programas de maestría profesional en \\ enfermeira
}

Recebido: 06/08/2021 | Revisado: 13/08/2021 | Aceito: 18/08/2021 | Publicado: 21/08/2021

\author{
Rejane Eleuterio Ferreira \\ ORCID: http://orcid.org/0000-0002-9328-174X \\ Universidade Federal do Rio de Janeiro, Brasil \\ E-mail: rejane_eleuterio@ hotmail.com \\ Cláudia Mara de Melo Tavares \\ ORCID: http://orcid.org/0000-0002-8416-6272 \\ Universidade Federal Fluminense, Brasil \\ E-mail: claudiamarauff@gmail.com
}

\begin{abstract}
Resumo
A aplicabilidade do conhecimento na prática é reconhecida mundialmente como um fator relevante para a melhoria da assistência prestada nos serviços de saúde. Logo, a translação do conhecimento tem se tornado uma exigência nas propostas dos cursos de mestrado profissional na área da Enfermagem. Objetivo: conhecer como a translação do conhecimento está sendo utilizada pelos programas de mestrado profissional na área da Enfermagem. Metodologia: trata-se de um estudo documental realizado no período de 2016 a 2019, nos sites dos programas de mestrado profissional na área da Enfermagem do Rio de Janeiro, norteado pela seguinte questão: como os programas de mestrado profissional na área da Enfermagem apresentam em sua proposta a perspectiva de translação de conhecimento? As informações acessadas foram: foco, princípios, objetivos, trabalho de conclusão e produto. Para realizar análise e discussão dos dados foram seguidas as instruções do método, fez-se também uma reflexão à luz da literatura científica nacional e internacional correlata. Resultados: numa análise global dos sites dos cursos, assim como os trabalhos de conclusão e os produtos, percebeu-se a inexistência de menção ou referência de modelo orientador ou estratégia para a translação do conhecimento nos programas de mestrado profissional na área da Enfermagem. No entanto, existem informações nas propostas que sugerem uma perspectiva translacional. Conclusão: no Brasil, a translação do conhecimento é tanto uma necessidade quanto um desafio, porém, para que haja uma melhor utilização de resultados de pesquisa, é necessário adaptar modelos teóricos de translação do conhecimento para o contexto brasileiro.

Palavras-chave: Educação de Pós-Graduação em Enfermagem; Pesquisa Científica e Desenvolvimento Tecnológico; Pesquisa Aplicada; Programas de Pós-Graduação em Saúde; Pesquisa Médica Translacional.
\end{abstract}

\begin{abstract}
Objective: to know how the translation of knowledge is being used in professional master's programs in the field of Nursing. Methodology: This is a documentary study carried out from 2016 to 2019, on the websites of professional master's programs in the field of Nursing in Rio de Janeiro, guided by the following question: How do professional master's programs in the field of Nursing present in your proposal the perspective of knowledge translation? The information accessed was: focus, principles, objectives, conclusion work and product. The analysis and discussion of the data were carried out following the instructions of the method, and making a reflection in the light of the related national and international scientific literature. Results: In a global analysis of the course websites, as well as the conclusion works and products, it was noticed that there was no mention or reference of a guiding model or strategy for the translation of knowledge in professional master's programs in the field of Nursing. However, there is information in the proposals that suggest a translational perspective. Conclusion: In Brazil, knowledge translation is both a necessity and a challenge, but in order to make better use of research results, it is necessary to adapt theoretical models of knowledge translation to the Brazilian context.

Keywords: Education, Nursing, Graduate; Scientific Research and Technological Development; Applied Research; Health Postgraduate Programs; Translational Medical Research.
\end{abstract}

\section{Resumen}

Objetivo: saber cómo se está utilizando la traducción del conocimiento en los programas de maestría profesional en el campo de la Enfermería. Metodología: Este es un estudio documental realizado entre 2016 y 2019, en los sitios 
web de programas de maestría profesional en el campo de la Enfermería en Río de Janeiro, guiado por la siguiente pregunta: ¿Cómo se presentan los programas de maestría profesional en el campo de la Enfermería en su propuesta la perspectiva de la traducción del conocimiento? La información a la que se accedió fue: enfoque, principios, objetivos, trabajo de conclusión y producto. El análisis y la discusión de los datos se llevaron a cabo siguiendo las instrucciones del método y haciendo una reflexión a la luz de la literatura científica nacional e internacional relacionada. Resultados: en un análisis global de los sitios web del curso, así como de los trabajos y productos de conclusión, se observó que no se mencionaba ni se hacía referencia a un modelo o estrategia orientadores para la traducción del conocimiento en los programas de maestría profesional en el campo de la Enfermería. Sin embargo, hay información en las propuestas que sugiere una perspectiva traslacional. Conclusión: en Brasil, la traducción del conocimiento es tanto una necesidad como un desafío, pero para hacer un mejor uso de los resultados de la investigación, es necesario adaptar los modelos teóricos de traducción del conocimiento al contexto brasileño.

Palabras clave: Educación de Posgrado em Enfermería; Investigación Científica y Desarrollo Tecnológico; Investigación Aplicada; Programas de Posgrado en Salud; Investigación en Medicina Traslacional.

\section{Introdução}

O mestrado profissional em Enfermagem é um programa stricto sensu cuja proposta é estimular o aluno a desenvolver estudos relacionados a problemas presentes no seu campo de atuação e formular soluções através do desenvolvimento de produções tecnológicas a serem incorporados em sua prática (Ferreira \& Tavares, 2020).

Embora todos os investimentos em pesquisa e capacitação dos profissionais de saúde, intervenções e programas eficazes nem sempre chegam aos usuários, ou seja, a simples criação de evidências, políticas e diretrizes não garante seu amplo uso ou adoção (Pereira et al., 2020; Thirsk, 2018). Acredita-se que alguns produtos são absorvidos nos serviços, porém ainda necessitam de desenho mais estruturado, considerando a incorporação na prática e consequentes benefícios para a equipe de saúde, pacientes e familiares. Mas este, dentre outros aspectos, raramente fazem parte do planejamento do estudo, o que daria a perspectiva translacional da pesquisa em Enfermagem (Crossetti \& Góes, 2017).

A Translação do Conhecimento (TC) é um conceito amplo, abrange todas as etapas entre a criação do conhecimento e sua aplicação para produzir resultados benéficos para a sociedade (Oelke, Lima \& Acosta, 2015). Trata-se de um processo importante para o desenvolvimento de novas intervenções, visto que ajuda a aplicar a ciência na prática, testando sua viabilidade e aceitação (Costa et al., 2019).

$\mathrm{O}$ interesse na TC tem aumentado nos últimos anos, pois se passou a entender como fundamental para o desenvolvimento de conhecimento relevante a disseminação e utilização de resultados de pesquisa. Até porque o conhecimento novo, por si só, não leva à grande aplicação nem efeito nos resultados de saúde (Oelke et al., 2015).

Até a última avaliação quadrienal (2013-2016) dos programas de mestrado profissional na área da Enfermagem, a TC se apresentava no documento de área da Coordenação de Aperfeiçoamento de Pessoal de Nível Superior (CAPES) como um estímulo à prática de Enfermagem e saúde, na perspectiva da prática baseada em evidência (Ministério da Educação, 2017).

Nos documentos mais recentes da área de Enfermagem na CAPES, a TC se destaca e se torna uma exigência ainda maior que esteja nas propostas dos cursos, embora não haja nenhum modelo de TC como referência (Ministério da Educação, 2019). Consequentemente, os programas têm atualizado as propostas dos cursos nos sites, buscando trazer a perspectiva de TC. Nesse sentido, este estudo tem como objetivo conhecer como a translação do conhecimento está sendo utilizada pelos programas de mestrado profissional na área da Enfermagem.

\section{Modelo de Translação de Conhecimento}

O principal conceito de TC foi definido pela agência federal Canadian Institutes of Health Research (CIHR, 2014, p. 2) como: "um processo dinâmico e interativo que inclui a síntese, disseminação, intercâmbio e aplicação eticamente sólida de conhecimento para melhorar a saúde, proporcionar produtos e serviços de saúde mais efetivos e fortalecer o sistema de 
saúde". Esse conceito foi adaptado por outras instituições, incluindo a Organização Mundial de Saúde (OMS), que entende a TC como um paradigma emergente que preenche a lacuna entre o conhecimento produzido e utilizado (Oelke et al., 2015).

O modelo de TC da CIHR é um dos mais utilizados no mundo, logo este estudo tomará como referência esse molde que apresenta duas abordagens de translação do conhecimento: TC integrada e TC no fim da concessão do projeto.

$\mathrm{Na}$ TC integrada, as partes interessadas e/ou os usuários estão envolvidos em todo o processo da pesquisa. Os interessados e os usuários são indivíduos ou grupos capazes de usar evidências geradas por pesquisa para tomar decisões fundamentadas sobre políticas de saúde, programas e práticas. Exemplos de usuários do conhecimento incluem profissionais de saúde, formuladores de políticas, educadores, gestores e administradores, líderes comunitários, e pacientes (Oelke et al., 2015; Silva, Alves \& Oliveira; 2019).

No processo de TC integrada, pesquisadores, partes interessadas e usuários trabalham juntos para moldar o processo de pesquisa. Nessa abordagem, todos os atores têm participação ativa no desenvolvimento do estudo, dentre elas: definição das questões de pesquisa, metodologia, envolvimento na coleta e interpretação dos dados, assim como na difusão dos resultados. Essa abordagem é também conhecida por termos como pesquisa colaborativa, pesquisa orientada para a ação e coprodução de conhecimento (CIHR, 2016).

A TC, no final da concessão, tem como objetivo disseminar os resultados e conhecimentos adquiridos durante a pesquisa para diversas audiências. Incluem tanto abordagens tradicionais (ex. apresentações em conferências, artigos científicos), como também atividades como vídeo, artigos na mídia, ou conjunto de ferramentas para uma área específica (CIHR, 2016).

Os estudos revelam que envolver usuários do conhecimento no início do processo garante uma pesquisa mais relevante e com domínio, o que irá facilitar a utilização dos resultados da investigação. Do mesmo modo, é importante, no processo de TC, monitorar o uso do conhecimento, determinando sua difusão para os grupos alvos de usuários e avaliar os principais resultados (Oelke et al., 2015).

A TC é desenvolvida a partir de um "modelo de conhecimento para ação", um processo que conceitua a relação entre criação e ação do conhecimento, com cada conceito composto por fases ou categorias ideais. No centro, existe um "funil" de criação de saberes que transmite a ideia de que o conhecimento precisa ser cada vez mais destilado antes de estar pronto para a aplicação. Em torno do funil, existe um ciclo que leva à sua implementação ou à sua aplicação. Ao contrário do funil, o ciclo de ação representa as atividades que podem ser necessárias para a aplicação do conhecimento (CIHR, 2016) (Figura1). 
Figura 1 - Processo do conhecimento para ação.

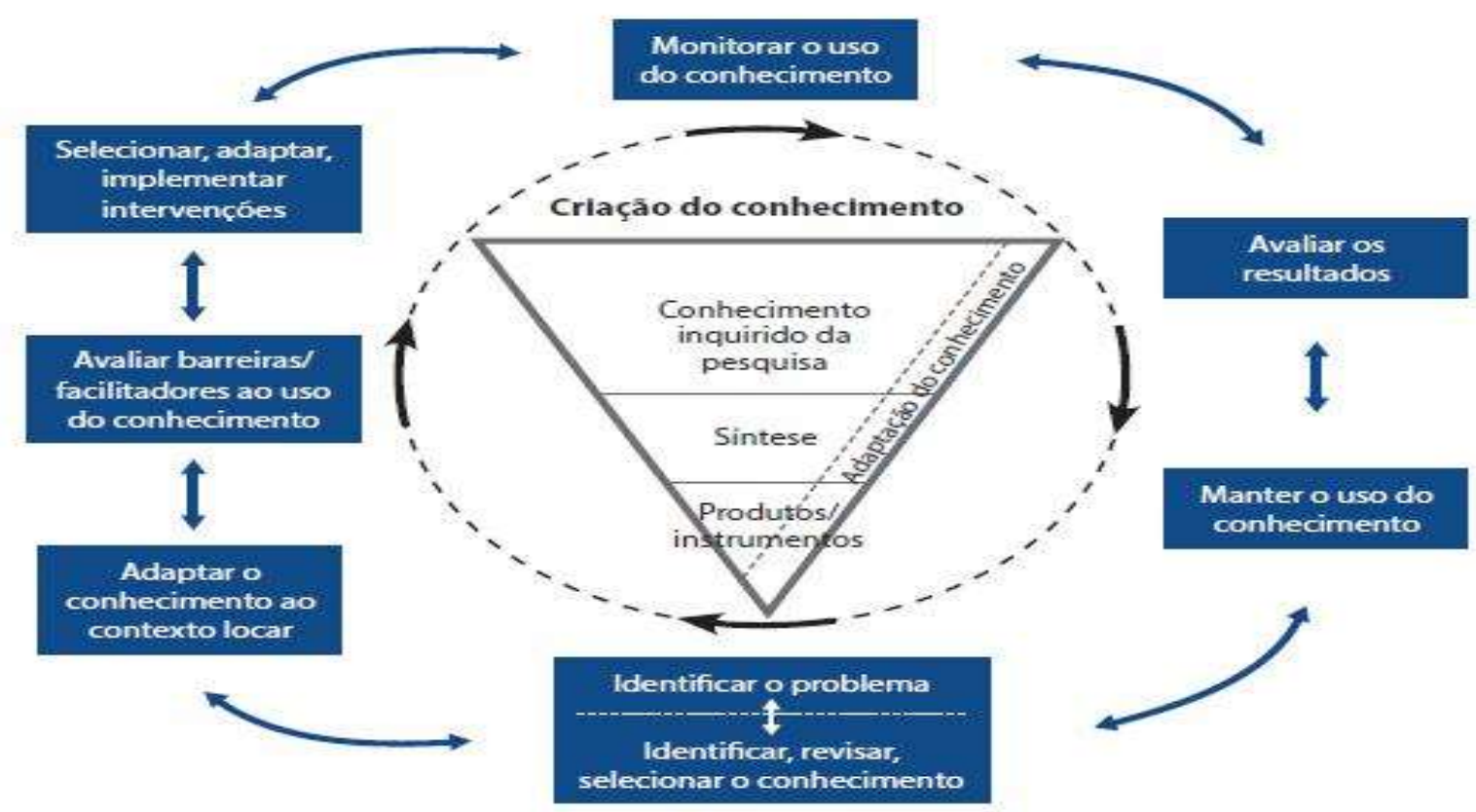

Fonte: Oelke et al. (2015).

A criação do conhecimento envolve três fases que aparecem na seguinte ordem, começando na parte superior do funil: pesquisa, síntese e produtos / ferramentas. O termo "conhecimento de adaptação" aparece ao longo do comprimento do funil à direita. As setas estão circulando o funil para ilustrar que as fases de criação de conhecimento podem influenciar as fases de ação em vários pontos ao longo do círculo (CIHR, 2016).

A aplicação do conhecimento em torno do funil consiste em sete fases, conectadas com setas bidirecionais: Na parte inferior do ciclo, aparece a orientação: identificar problema. No sentido horário, as fases restantes, são: adaptar ao contexto local; avaliar barreiras ao uso; selecionar, adaptar, implementar intervenções; monitorar o uso; avaliar resultados; e sustentar o uso do conhecimento (CIHR, 2016).

O processo pode se iniciar com a criação do conhecimento, importante fase que sintetiza os saberes e o tornam mais úteis para os usuários finais. O ciclo de ação enfoca atividades necessárias para aplicar o conhecimento nos sistemas de saúde. Estão incluídos desde a identificação de um problema até o planejamento para sustentabilidade do uso do conhecimento na prática e na formulação de políticas. É crucial adaptar o conhecimento para o contexto local, avaliar barreiras e facilitadores para seu uso e dirigir o conhecimento para as necessidades das pessoas que irão usá-lo, considerando o orçamento (Oelke et al., 2015).

\section{Metodologia}

Trata-se de uma pesquisa documental de abordagem qualitativa, que possibilita a observação do processo de maturação ou de evolução de conceitos, conhecimentos, comportamento, prática, entre outros (Andrade, Schmitt, Storck, Piccoli, \& Ruoff, 2018).

A pesquisa foi realizada nos sites dos programas de mestrado profissional na área da Enfermagem do estado do Rio de Janeiro. O critério de inclusão foi: site ativo que apresente informações do curso de mestrado profissional. Critério de exclusão: site desatualizado e/ou que não contenha informações referente à proposta do curso, trabalho de conclusão e produto. 
A coleta de dados foi realizada no período de 2016 a 2019, com objetivo de acompanhar as mudanças no conteúdo do site para atender a novas exigências da CAPES referentes à translação do conhecimento. Foram analisados os conteúdos do site de três programas de mestrado profissional na área da Enfermagem no estado do Rio de Janeiro.

Coletaram-se os dados por meio de um roteiro estruturado, com base nos elementos do modelo de TC da CIRH (2016), norteado pela seguinte questão: como os programas de mestrado profissional na área da Enfermagem apresentam em sua proposta a perspectiva de translação de conhecimento?

As informações obtidas tiveram as seguintes variáveis: foco, princípios, objetivos, trabalho de conclusão de curso e produto. Organizamos esses conteúdos em um grande quadro comparativo que foi sendo alimentado com as principais mudanças no decorrer do corte temporal desta pesquisa.

Após a seleção e análise preliminar dos documentos, procedeu-se à análise dos dados. Nessa etapa de análise dos documentos, reelaboraram-se conhecimentos e formas de compreender o fenômeno em estudo, determinando tendências, tendo em conta a temática ou o questionamento inicial e, na medida do possível, fazendo a inferência (Andrade et al., 2018). Discutiram-se os resultados, seguindo as instruções do método, e fazendo uma reflexão à luz da literatura científica nacional e internacional correlata.

Vale ressaltar que a pesquisa documental utilizou informações de domínio público e, por não envolver seres humanos diretamente na coleta dos dados, não houve exigência de aprovação do Comitê de Ética em Pesquisa (Conselho Nacional de Saúde, 2016). Para realizarmos a análise dos programas de mestrado profissional na área da Enfermagem, mantivemos o anonimato das instituições.

\section{Resultados}

Numa análise global dos sites dos cursos, assim como os trabalhos de conclusão e os produtos, percebeu-se a inexistência de menção ou referência de modelo orientador ou estratégia para a translação do conhecimento nos programas de mestrado profissional na área da Enfermagem.

Verificou-se que os programas atualizaram recentemente as informações presentes nos sites, dentre elas: atualizações das propostas dos cursos, forma de apresentação dos trabalhos de conclusão que estavam em um modelo acadêmico de dissertação e a criação de um espaço especifico com destaque para os produtos desenvolvidos no âmbito do mestrado profissional na área da Enfermagem.

Nos sites dos programas analisados, fala-se muito de inserção social, mas ainda é discreta a apresentação de como ocorre de fato, o que não permite uma dimensão de impacto. O que se percebe em comum é a busca de parcerias e convênios com instituições de saúde e de ensino públicas e privadas.

Um curso específico tem em sua proposta a participação dos gestores nas defesas. Essa estratégia é utilizada por meio de seminários que contam com a participação de todos os professores do programa, docentes externos e gestores na primeira etapa do curso, com a defesa do projeto de pesquisa, mas que pode se estender a outras etapas do processo de formação. O objetivo é desenvolver ações coletivas, trabalhos cooperativos e em grupo no processo de construção do produto. Outras experiências desse curso são: utilização de metodologias ativas como forma de garantir a indissociabilidade entre teoria e prática e integração com a graduação e Programa de Ensino pelo Trabalho (PET-Saúde) do Ministério da Saúde.

Ao analisar esses itens da proposta do curso sob perspectiva de abordagem da TC segundo a CIHR, podemos dizer que o programa se aproxima de uma proposta de translação do conhecimento apoiada em uma abordagem de TC integrada, processo em que pesquisadores, partes interessadas e usuários trabalham juntos para moldar o processo de pesquisa. Nessa abordagem todos os atores têm participação ativa no desenvolvimento do estudo. 
Os outros dois programas não apresentam em sua proposta uma perspectiva colaborativa na construção do produto, o que deduz a uma aproximação da abordagem de TC para fins de concessão do produto. Ou seja, a busca por colaboradores está mais intencionada na difusão do conhecimento desenvolvido no decorrer da pesquisa, do que nas decisões do processo de construção.

Temos como exemplo um curso que tem entre seus objetivos "atender à orientação de produção de inovação tecnológica (dura, leve-dura, leve) no espaço hospitalar a partir de evidências identificadas". Ou seja, o produto é desenvolvido a partir das evidências científicas.

No outro curso, a indicação de uma abordagem de TC para o fim da concessão do projeto esta presente em vários objetivos, dentre eles "promover a apropriação e a transferência do conhecimento científico e inovação para a prática profissional, tendo em vista as demandas sociais, profissionais e do mercado de trabalho" e também na construção de um perfil de aluno para que ele tenha um potencial empreendedor e competitivo no mercado de trabalho.

\section{Discussão}

A perspectiva do mestrado profissional de realizar interação entre conhecimento científico e prática profissional também é conhecida como a translação do conhecimento. Este termo é relativamente novo e permanece pouco conhecido no Brasil.

Ele é originário da teoria da translação utilizada no campo da linguística e da comunicação e vem sendo adotado em diversos campos, inclusive no da saúde, desde meados da década de 1970, porém começou a ser amplamente usado a partir da década de 1990 (Abreu, Santos, Cardoso \& Artmann, 2017).

O conceito de TC é parte desse novo cenário epistemológico e parece estar consonante com os paradigmas científicos contemporâneos, na medida em que suplanta as fronteiras disciplinares do conhecimento e instiga o diálogo entre diferentes saberes (Colombo, Anjos \& Antunes, 2019).

Assim, o encontro entre o conhecimento científico e o conhecimento fruto da experiência e da vivência no dia a dia permite o desenvolver a compreensão e o discernimento entre universos inicialmente estranhos, que, de modo gradual, reconfiguram-se a partir dessa interação compartilhada (Barbosa \& Pereira Neto, 2017).

É importante ressaltar que a TC difere dos movimentos de transferência, pois não estabelece fluxos unidirecionais que partem do pesquisador para o usuário. Pelo contrário, ela pretende ser multidirecional, agindo a partir da troca e diálogo entre diferentes saberes, o que inclui o saber científico e o popular (Barbosa \& Pereira Neto, 2017).

Tal ressalva é importante, pois ainda sofremos forte influência de um paradigma dominante, e a TC, por ser uma temática contemporânea, é entendida algumas vezes como uma ação de transferência unilateral. No entanto, não basta a construção de conhecimento novo ou sua divulgação. Faz-se necessário promover sua utilização na prática e, para que isso ocorra, é necessário um movimento multidirecional com co-construção de todos os autores envolvidos.

Contudo, colocar novos conhecimentos em prática é um processo complexo que depende do preparo técnico e da habilidade de quem vai usá-lo, de fatores organizacionais de suporte, do envolvimento contínuo dos potenciais usuários do conhecimento, das parcerias estabelecidas, do contexto institucional e da existência de mediadores do conhecimento (Bezerra, Felisberto, Costa, \& Hartz, 2019).

O desenvolvimento de um plano de TC é essencial quando se inicia um novo projeto de pesquisa. Um estudo de revisão evidenciou mais de 28 diferentes modelos de TC. Entre os elementos em comum, estão: identificação do problema e estabelecimento de canais de comunicação entre usuários e pesquisadores, desenvolvimento e seleção do tipo de pesquisa, análise do contexto, realização de atividades ou intervenções para translação de conhecimento e aplicação dos resultados na 
prática (Crossetti \& Góes, 2017).

O país que mais utiliza a TC é o Canadá, inclusive para auxiliar no processo de tomada de decisões. Aperfeiçoar o sistema de saúde e melhorar o bem-estar dos indivíduos, a partir de um processo dinâmico de síntese, disseminação, troca e aplicação ética do conhecimento, são os principais objetivos da TC para esse país. Para tanto, é constituído um complexo sistema de interações entre pesquisadores e usuários (Barbosa \& Pereira Neto, 2017).

Nos últimos anos, aumentou o interesse pela TC, pois a aplicabilidade do conhecimento na prática é reconhecida mundialmente como um fator relevante para o fortalecimento do sistema de saúde e melhoria da assistência prestada (Silva et al., 2019; Lupatini et al., 2019). É oportuno enfatizar que a pesquisa translacional é útil para tomadores de decisão se beneficiarem dos conhecimentos científicos produzidos, avaliando para além da eficácia das intervenções (Silva et al., 2019; Andrade \& Pereira, 2019). Na enfermagem, uma pesquisa revelou que a translação do conhecimento pode fortalecer a implementação o processo de enfermagem e a melhoria da assistência em enfermagem (Oliveira, Silvino \& Souza, 2021).

No Brasil, a TC é tanto uma necessidade quanto um desafio. Na agenda nacional de prioridades de pesquisa em saúde, a TC não aparece no cenário da pós-graduação stricto sensu, aparece apenas uma vez no documento da área da CAPES que avalia os programas de mestrado, como um dos desafios que permanecem presentes para o quadriênio vigente, desafio este descrito como "estimular a translação de conhecimento para a prática de Enfermagem e saúde, na perspectiva da prática baseada em evidência" (Ministério da Educação, 2017, p. 9).

No entanto, apesar dos 40 anos de pesquisa em políticas baseadas em evidências, muitos são os desafios para os pesquisadores aumentarem o uso do conhecimento produzido e desses estudos serem usados como formuladores de políticas (Bezerra et al., 2019).

O documento de área publicado recentemente pela CAPES, recomenda que: "O planejamento de cada programa precisa estar voltado ao desenvolvimento de boas práticas em saúde, com vistas à translação do conhecimento e sua aplicação em serviços de saúde" (Ministério da Educação, 2019, p. 13). Essa recomendação tem sido atendida por alguns programas da área da Enfermagem, pois, nos sites, já é possível identificar na proposta de alguns cursos a perspectiva da translação do conhecimento, contudo inexiste a menção ou referência de modelo orientador ou estratégia para a translação do conhecimento.

A literatura internacional descreve as principais barreiras para a implementação da TC no Brasil, dentre elas: falta de familiaridade; dificuldades em identificar problema de pesquisa relevante; pouco envolvimento dos principais interessados e falta de parceria entre pesquisadores e usuários do conhecimento no processo de investigação; falta de concordância com evidências ou resultados de estudos; falta de percepção do valor e benefício de mudar a prática, falta de praticidade; falta de tempo, habilidade e motivação; baixos orçamentos para pesquisa e pouco enfoque em TC pelas políticas de agência de financiamento (Oelke et al., 2015; Crossetti \& Góes, 2017; Bezerra et al., 2019).

O cenário de pesquisa atual do Brasil sofre forte influência de um modelo acadêmico, em que, tradicionalmente, a utilização de resultados de pesquisa na prática é lenta, levando frequentemente muitos anos, de forma que a inovação na prestação de serviços de saúde é difícil e alguns resultados de pesquisa obsoletos, formando uma lacuna entre pesquisa, prática e políticas de saúde (Oelke et al., 2015). Essa influência de um modelo acadêmico estava presente inclusive nos trabalhos de conclusão do mestrado profissional na área da Enfermagem que se apresentavam todos no formato de dissertação. Esse formato é exclusivo dos cursos acadêmicos, posto que o mestrado profissional tem outras possibilidades de apresentação de trabalho de conclusão, além da dissertação (Ferreira \& Tavares, 2020). As atualizações recentes nos sites analisados já apresentam uma mudança na apresentação dos trabalhos, que deixam de ser exclusivamente no formato de dissertação.

$\mathrm{O}$ mestrado profissional é criado com uma perspectiva de atender à demanda do mercado de trabalho e atender às necessidades da população, mas os estudos apontam que as pesquisas desenvolvidas no âmbito do mestrado profissional na área da Enfermagem geram conhecimento a partir da prática, porém, os estudos são restritos e, em sua maioria, há ações 
investigativas que não resultam em mudanças imediatas, ainda que produzam resultados que possibilitam intervenções posteriores (Ferreira, Tavares \& Kebian, 2018). Isso ocorre porque o processo de implantação do resultado na prática raramente faz parte do planejamento da investigação, o que configuraria uma perspectiva translacional da pesquisa em Enfermagem.

São muitas as barreiras a serem enfrentadas para que haja troca e aplicação do conhecimento com vistas a potencializar os benefícios decorrentes de inovações para fortalecer sistemas de saúde e melhoria da saúde das populações, auxiliando, como no Canadá, a tomada de decisão do nosso país. Para melhor utilização de resultados de pesquisa, é necessário adaptar modelos teóricos de TC para o contexto brasileiro. Essa definição de um modelo de TC poderia ser indicada pela própria CAPES e, caso fosse adotada pelos programas de mestrado profissional, facilitaria uma avaliação dos cursos.

\section{Considerações Finais}

O presente estudo teve como objetivo conhecer como a translação do conhecimento está sendo utilizada nos programas de mestrado profissional na área da Enfermagem. Os resultados apontaram a inexistência de menção ou referência de modelo orientador ou estratégia para a translação do conhecimento nas propostas dos programas, trabalhos de conclusão e produtos disponíveis nos sites dos cursos analisados.

Embora aparentemente não haja a adoção de um modelo de translação do conhecimento nas propostas das pesquisas desenvolvidas nos programas de mestrado profissional na área da Enfermagem, foi possível identificar que os cursos fizeram atualizações recentes no site e apresentaram em suas propostas informações que sugerem uma perspectiva translacional. Essas atualizações parecem ter relação com as novas recomendações da CAPES para que haja TC nas propostas dos programas.

No entanto, as atualizações que visam a uma perspectiva translacional nas pesquisas desenvolvidas no mestrado profissional na área da Enfermagem, ainda são discretas e carecem de um modelo de TC. No Brasil, a TC é tanto uma necessidade quanto um desafio. Porém, para que haja uma melhor utilização de resultados de pesquisa, é necessário adaptar modelos teóricos de TC para o contexto brasileiro. Nesse sentido, faz-se necessário desenvolver novos estudos que avalie como as outras áreas de mestrado profissional têm avançado para desenvolver a translação do conhecimento em seus cursos e que avalie a implantação de um modelo de translação de conhecimento em programas de mestrado profissional e seus impactos.

\section{Referências}

Abreu, D. M. F.; Santos, E. M.; Cardoso, G. C. P. \& Artmann, E. (2017). Usos e influências de uma avaliação: translação de conhecimento? Saúde em Debate, 41(N. Especial), 302-316. http://docvirt.com/asp/acervo_cebes.asp?Bib=SAUDEDEBATE\&PASTA=V.41\%2C+N.112+-+jan.\%2Fmar.\%2F2017 \&pesq $=\& x=68 \& y=4$.

Andrade, K. R. C. de., \& Pereira, M. G. (2020). Knowledge translation in the reality of Brazilian public health. Revista de Saúde Pública, 54, 72. https://doi.org/10.11606/s1518787.2020054002073.

Andrade, S. R.; Schmitt, M. D.; Storck, B. C .; Piccoli, T. \& Ruoff, A. B. (2018). Documentary analysis in nursing theses: data collection Techniques and research methods. Cogitare Enferm, 23(1):e53598. https://revistas.ufpr.br/cogitare/article/view/53598/pdf_en. doi: 10.5380/ce.v23i1.53598.

Barbosa, L. \& Pereira Neto, A. (2017). Ludwik Fleck (1896-1961) e a translação do conhecimento: considerações sobre a genealogia de um conceito. Saúde em Debate, 41(N. Especial), 317-329. http://docvirt.com/asp/acervo_cebes.asp?Bib=SAUDEDEBATE\&PASTA=V.41\%2C+N.112++ jan.\%2Fmar.\%2F2017\&pesq $=\& \mathrm{x}=68 \& \mathrm{y}=4$.

Bezerra, L. C. A.; Felisberto, E.; Costa, J. M. B. S. \& Hartz, Z. (2019). Translação do Conhecimento na qualificação da gestão da Vigilância em Saúde: contribuição dos estudos avaliativos de pós-graduação. Physis: Revista de Saúde Coletiva, 29(1): e290112, 1-33. https://www.scielo.br/scielo.php?script=sci_arttext\&pid=S0103-73312019000100611. doi: 10.1590/s0103-73312019290112.

Canadian Institutes of Health Research. (2014). More about knowledge translation at CIHR: knowledge translation definition. http://www.cihrirsc.gc.ca/e/39033.html.

Canadian Institutes of Health Research. (2016). More About Knowledge Translation at CIHR: Long Descriptions. http://www.cihr-irsc.gc.ca/e/29418.html. 
Research, Society and Development, v. 10, n. 11, e07101119168, 2021

(CC BY 4.0) | ISSN 2525-3409 | DOI: http://dx.doi.org/10.33448/rsd-v10i11.19168

Colombo, I. M.; Anjos, D. A. S. \& Antunes, J. R. (2019). Pesquisa translacional em ensino: uma aproximação. Educação Profissional e Tecnológica em Revista, 3(1), 51-70. https://ojs.ifes.edu.br/index.php/ept/article/view/377/338.

Conselho Nacional de Saúde (Brasil). Resolução no 510, de 7 de abril de 2016. Diário Oficial da União, 98, Seção 1, 44-46.

Costa, P.; Palombo, C. N. T.; Silva, L. S.; Silva, M. T.; Mateus, L V J \& Buchhorn, S. M. M. (2019). Ações de extensão universitária para translação do conhecimento sobre desenvolvimento infantil em creches: relato de experiência. Rev. esc. Enferm., 53:e03484, 1-8. https://www.scielo.br/pdf/reeusp/v53/1980-220X-reeusp-53-e03484.pdf. doi: 10.1590/s1980-220x2018020603484.

Crossetti, M. G. O. \& Góes, M. G. O. (2017). Translação do conhecimento: um desafio para prática de enfermagem. Rev. Gaúcha Enferm., 38 (2):e74266, 1-2. https://www.scielo.br/pdf/rgenf/v38n2/0102-6933-rgenf-1983-144720170274266.pdf. doi: 10.1590/1983-1447.2017.02.74266.

Ferreira, R. E. \& Tavares, C. M. M. (2020). Análise da produção tecnológica de três programas de mestrado profissional na área da Enfermagem. Rev. LatinoAm. Enfermagem, 28:e3276, 1-8. https://www.scielo.br/pdf/rlae/v28/pt_0104-1169-rlae-28-e3276.pdf. doi: 10.1590/1518-8345.3916.3276.

Ferreira, R. E.; Tavares, C. M. M. \& Kebian, L.V.A. (2018). Scientific production related to the professional master in nursing. J Nurs UFPE Online, 12(3), 763-771. https://periodicos.ufpe.br/revistas/revistaenfermagem/article/view/230612/28043. doi: 10.5205/1981-8963-v12i3a230612p763-771-2018.

Lupatini, E. O., Barreto, J. O. M., Zimmermann, I. R., \& Silva, E. N. (2019, novembro). Medicamentos e pesquisa translacional: etapas, atores e políticas de saúde no contexto brasileiro. Saúde em Debate, 43(spe2), 181 - 199. https://doi.org/10.1590/010311042019S214

Ministério da Educação (Brasil). (2016). Coordenação de Aperfeiçoamento de Pessoal de Nível Superior. Documento de Área - Área 20 - Enfermagem. 1-45.

Ministério da Educação (Brasil). (2019). Coordenação de Aperfeiçoamento de Pessoal de Nível Superior. Documento de Área - Área 20 - Enfermagem. 1-23.

Oelke, N. D.; Lima, M. A. D. S. \& Acosta, A. M. (2015). Translação do conhecimento: traduzindo pesquisa para uso na prática e na formulação de políticas. Rev. Gaúcha Enferm., 36 (3), 113-117. https://seer.ufrgs.br/RevistaGauchadeEnfermagem/article/view/55036/35070. doi: 10.1590/1983-1447.2015.03.55036.

Oliveira, A. L. G. de ;Silvino, Z. R. .; Souza, C. J. de. (2021). Translation of knowledge in the Implementation of the nursing process in a neonatal

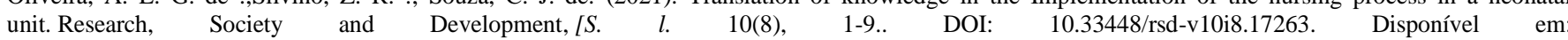
https://rsdjournal.org/index.php/rsd/article/view/17263. Acesso em: 6 aug. 2021.

Pereira, R P. G.; Schneider, L. R.; Ferraz, L. (2020). A translação do conhecimento: as barreiras dos enfermeiros de cuidados de saúde primários. Revista $\begin{array}{llllll}\text { Científica } & \text { Internacional } & \text { RevSALUS. } & \text { Suplemento } & 2 & \text { setembro } \\ \end{array}$ epositorium.uminho.pt/bitstream/1822/68710/1/RACS_Suplemento_n2_Setembro2020_AF_P101.pdf

Silva, J. C.; Alves, C. K. A. \& Oliveira, S. R. A. (2019). Cartão de Evento-Crítico: ferramenta analítica para translação do conhecimento. Saúde em Debate, 43(N. Especial 2), 10-18. http://revista.saudeemdebate.org.br/sed/issue/view/32.

Thirsk J. (2018) Knowledge translation. Nutrition \& Dietetics 75: 341-44. 